\title{
Characteristic and Surge Impedance Variation Impact on Transmission Line Performance
}

\author{
Muhyaddin J. H. Rawa \\ Department of Electrical and Computer Engineering, King Abdulaziz University, Saudi Arabia
}

\begin{tabular}{lll}
\hline \hline Article Info & ABSTRACT \\
\cline { 1 - 2 } Article history: & $\begin{array}{l}\text { Modeling of power systems is essential to perform various network analyses. } \\
\text { Voltage regulation, line losses and transmission line efficiency are greatly } \\
\text { affected by transmission line parameters. Hence, accurate modeling of } \\
\text { Received Jan 19, 2018 } \\
\text { Revised Mar 28, 2018 } \\
\text { Accepted Apr 5, 2018 }\end{array}$ & $\begin{array}{l}\text { transmission line is required. The aim of this paper is to study the impact of } \\
\text { characteristic and surge impedances on voltage profile, voltage regulation } \\
\text { and transmission line efficiency. }\end{array}$ \\
\hline Keyword: &
\end{tabular}

Characteristic impedance

Surge impedance

Transmission line modeling

Voltage regulation

Copyright $\odot 2018$ Institute of Advanced Engineering and Science. All rights reserved.

\section{Corresponding Author:}

Muhyaddin J. H. Rawa,

Department of Electrical and Computer Engineering,

King Abdulaziz University,

P.O. Box 80204, Jeddah 21589, Saudi Arabia.

Email: mrawa@kau.edu.sa

\section{INTRODUCTION}

Modeling of power systems is essential in order to perform various power system analyses such as load flow studies, short circuit calculations, contingency analyses and transient analyses. Electric power generation, transmission and distribution networks need to be modeled carefully for accurate results. This paper aims to study the effect of characteristic and surge impedance on transmission line performance; therefore, accurate modeling of transmission lines are considered.

In general, a "per-phase" model is used to represent equivalent circuits of transmission lines with appropriate circuit parameters. Depending on the length of the line, three different models are considered. Short transmission line model for length less than $80 \mathrm{~km}(50 \mathrm{mile})$ long, medium transmission line model for length between $80 \mathrm{~km}$ (50 mile) and $240 \mathrm{~km}$ (150 mile) long and long transmission line model for transmission lines longer than $240 \mathrm{~km}(150 \mathrm{mi})$ [1]-[5].

All lines are made up of distributed series resistance and inductance as well as shunt capacitance and conductance. They are called line parameters and denoted as R, L, C, and G. The most important parameters are R, L and C as they impact voltage drop, line losses and overall transmission line efficiency [6]-[9].

The paper is organized as follows. Short introduction regarding transmission line is given in Section 1. Modeling of transmission lines is discussed in Section 2. Section 3 describes transmission line voltage regulation and efficiency. The effects of characteristic impedance on transmission line performance are discussed in Section 4. The impacts of surge impedance on transmission line performance are introduced in Section 5. Summary and conclusion are discussed in Section 6. 


\section{MODELLING OF TRANSMISSION LINES}

The generalized circuit constants commonly known as ABCD constant are used to draw the relationships between sending- and receiving-end voltages and currents as follows [2]-[6], [10]-[14].

$$
\begin{aligned}
& I_{S}=C V_{R}+D I_{R} \\
& V_{S}=A V_{R}+B I_{R}
\end{aligned}
$$

It can be written in a matrix form as follows.

$$
\left[\begin{array}{c}
V_{S} \\
I_{S}
\end{array}\right]=\left[\begin{array}{ll}
A & B \\
C & D
\end{array}\right]\left[\begin{array}{c}
V_{R} \\
I_{R}
\end{array}\right]
$$

The total series impedance of the line per phase is given by multiplying the series line impedance per unit length by the total length of the line.

$$
Z=z \ell=(r+j \omega L) \ell
$$

Where

$\mathrm{z}$ is the series line impedance per unit length.

$r$ is the per-phase resistance per unit length.

$\mathrm{L}$ is the per-phase inductance per unit length.

$\omega_{\text {is the angular frequency. }}$

$\ell$ is the line length.

For short transmission lines, the impact of the line capacitance is negligible. Notwithstanding, for medium and long lines, the capacitance of the transmission lines should be considered.

$$
Y=y \ell=(g+j \omega C) \ell
$$

Where

$\mathrm{g}$ is the shunt conductance per unit length.

$\mathrm{C}$ is the line to neutral capacitance per unit length.

$\mathrm{Y}$ is the total shunt admittance per phase to neutral.

The ABCD constant for long transmission lines are given by:

$$
A=\cosh (\gamma)
$$

$$
B=Z_{c} \sinh (\chi)
$$

$$
C=\frac{1}{Z_{c}} \sinh (x)
$$

$$
D=\cosh (\gamma)
$$

$$
\gamma=\alpha+j \beta=\sqrt{z y}
$$

Where

$\gamma$ is the propagation constant 
$\alpha$ is the attenuation constant

$\beta$ is the phase constant

The characteristic impedance $\mathrm{Zc}$ is given by:

$$
Z_{c}=\sqrt{\frac{z}{y}}
$$

Therefore,

$$
\begin{aligned}
& V_{S}=\cosh \gamma\left(V_{R}+Z_{c} \sinh \gamma I_{R}\right. \\
& I_{S}=\frac{1}{Z_{c}} \sinh \gamma \ell V_{R}+\cosh \gamma I_{R}
\end{aligned}
$$

By rearranging the two equations and using the identity:

$$
\tanh \frac{\chi}{2}=\frac{\cosh x-1}{\sinh x}
$$

One can obtain the equivalent $\pi$ model for long transmission lines as:

$$
\begin{aligned}
& V_{S}=\left(1+\frac{Z^{\prime} Y^{\prime}}{2}\right) V_{R}+Z^{\prime} I_{R} \\
& I_{S}=Y^{\prime}\left(1+\frac{Z^{\prime} Y^{\prime}}{4}\right) V_{R}+\left(1+\frac{Z^{\prime} Y^{\prime}}{2}\right) I_{R}
\end{aligned}
$$

Where

$$
\begin{aligned}
& Z^{\prime}=Z_{c} \sinh \chi=Z \frac{\sinh \chi}{\gamma} \\
& \frac{Y^{\prime}}{2}=\frac{1}{Z_{c}} \tanh \frac{\chi}{2}=\frac{Y}{2} \frac{\tanh \chi / 2}{\chi / 2}
\end{aligned}
$$

Figure 1 shows the equivalent $\pi$ model for long transmission line [2].

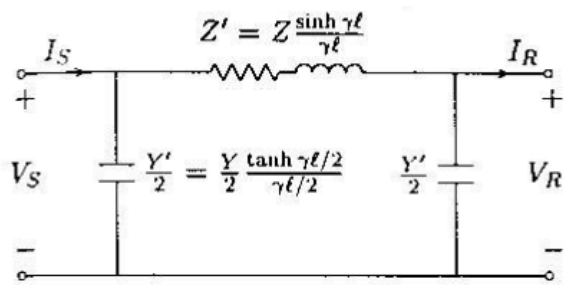

Figure 1. Equivalent $\pi$ model for long transmission line

\section{TRANSMISSION LINE VOLTAGE REGULATION AND EFFICIENCY}

Transmission-line voltages vary depending on load condition. During light load conditions, the voltage is high while it is low during heavy load conditions. Voltage regulation refers to the change in line 
voltage as line loading varies from no-load to full-load. Voltage regulation is a measure of a line voltage drop and can be calculated using the following equation [2], [6].

$$
\left|V_{R(N L)}\right|=\frac{\left|V_{S}\right|}{|A|}
$$

At no-load, IR=0; therefore, from (12).

$$
\% V R=\frac{\left|V_{R(N L)}\right|-\left|V_{R(F L)}\right|}{\left|V_{R(F L)}\right|} \times 100
$$

The sending-end and receiving-end powers are:

$$
\begin{aligned}
& S_{S(3 \phi)}=3 V_{S} I_{S}^{*}=P_{S(3 \phi)}+j Q_{S(3 \phi)} \\
& S_{R(3 \phi)}=3 V_{R} I_{R}^{*}=P_{R(3 \phi)}+j Q_{R(3 \phi)}
\end{aligned}
$$

Where

$$
\begin{aligned}
& S_{S(3 \phi)} \text { is the three phase sending-end complex power } \\
& S_{R(3 \phi)} \text { is the three phase receiving-end complex power } \\
& P_{S(3 \phi)} \text { is the three phase sending-end active power } \\
& P_{R(3 \phi)} \text { is the three phase receiving-end active power } \\
& Q_{S(3 \phi)} \text { is the three phase sending-end reactive power } \\
& Q_{R(3 \phi)} \text { is the three phase receiving-end reactive power }
\end{aligned}
$$

Transmission line efficiency $(\eta)$ is given by [2], [3].

$$
\eta=\frac{P_{R(3 \phi)}}{P_{S(3 \phi)}}
$$

\section{RESULTS AND DISCUSSION}

In order to study the effects of the characteristic impedance on the transmission line performance a $380 \mathrm{kV}, 60 \mathrm{~Hz}, 300 \mathrm{~km}$ long transmission line is considered. The line transmits a $1000 \mathrm{MVA}$ at 0.8 power factor. The line parameters are $\mathrm{r}=0.016 \Omega / \mathrm{km}, \mathrm{L}=0.97 \mathrm{mH} / \mathrm{km}, \mathrm{C}=0.0115 \mu \mathrm{F} / \mathrm{km}$ and $\mathrm{g}=0 . \mathrm{Z}_{\mathrm{c}}$ increases as $\mathrm{r}$ and $\mathrm{L}$ increase in steps of $5 \%$.

\subsection{Effects of characteristic impedance on transmission line performance}

The relationship between the characteristic impedance and receiving-end voltage is shown in Figure 2. It can be shown that increasing the characteristic impedance from about 285.4 to $346.3 \Omega$ increases the receiving-end voltage from 278.5 to $330.6 \mathrm{kV}$.

Figure 3 illustrates that increasing the characteristic impedance from about 285.4 to $346.3 \Omega$ improves the voltage regulation by approximately $39 \%$.

It can be seen from Figure 4 that increasing the characteristic impedance from about 285.4 to 346.3 $\Omega$ reduces the transmission line efficiency from 95.5 to $93.5 \%$. 


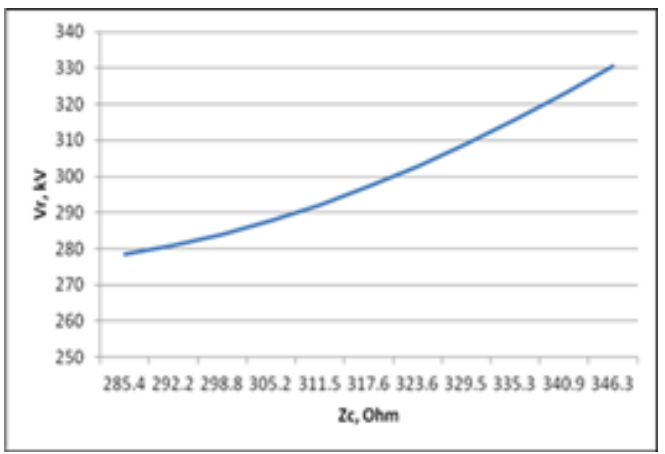

Figure 2. Characteristic impedance versus receivingend voltage

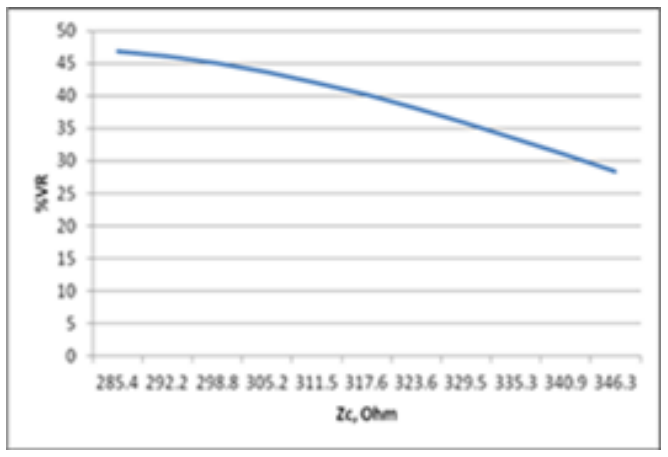

Figure 3. Characteristic impedance versus percentage voltage regulation

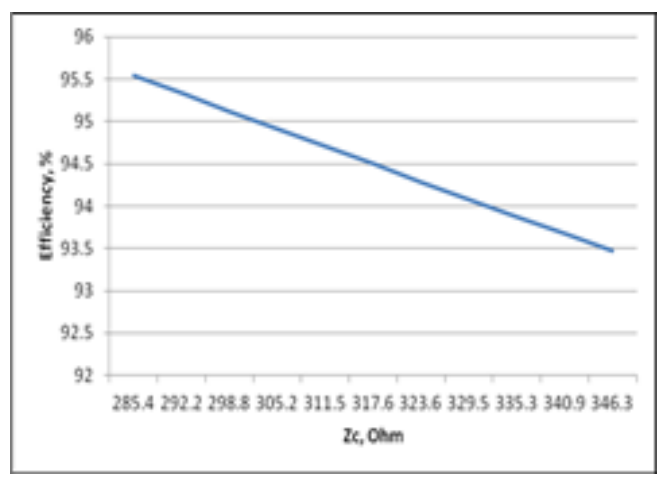

Figure 4. Characteristic impedance versus transmission line efficiency

\subsection{Effects of surge impedance on transmission line performance}

For a lossless transmission line, $\mathrm{r}=0$ and $\mathrm{g}=0$. Hence, the characteristic impedance $\mathrm{Z}_{\mathrm{c}}$ becomes:

$$
Z_{c}=\sqrt{\frac{L}{C}}
$$

Which is commonly known as the surge impedance. Figure 5 illustrates that increasing the surge impedance from 290.4 to $355.7 \Omega$ also increases the receiving-end voltage from 291.1 to 346 .

Figure 6 proves that increasing the surge impedance from 290.4 to $355.7 \Omega$ decreases the percentage voltage regulation by about $43.8 \%$.

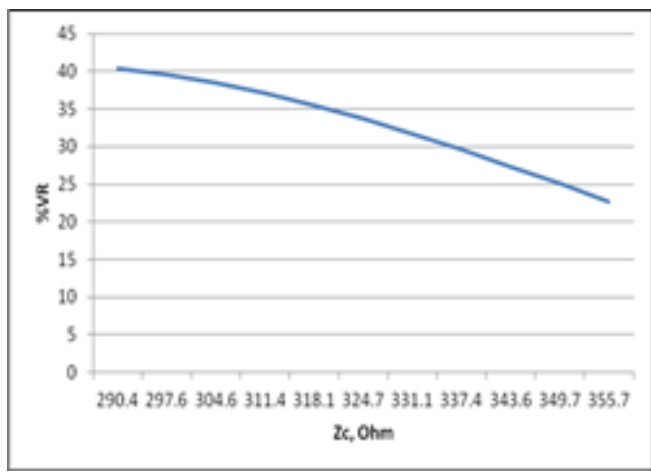

Figure 5. Surge impedance versus receiving-end voltage

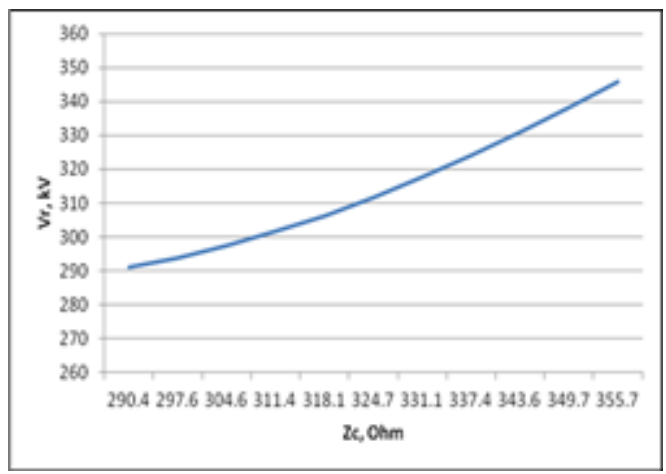

Figure 6. Characteristic impedance versus percentage voltage regulation 
As $r=0$ and $g=0$, there is no power losses in the transmission line. Hence, $P_{r}=P_{s}$ and the transmission line efficiency in this case is always $100 \%$.

\section{CONCLUSION}

Transmission lines constitute an integral part of power systems and the accuracy of many analyses such as load flow studies, short circuit calculations, contingency and transient analyses depend on the accurate modeling of the transmission lines. It has been proved in this paper that increasing the characteristic impedance or the surge impedance increases the receiving-end voltage. Furthermore, increasing the characteristic impedance or the surge impedance decreases the percentage voltage regulation. Moreover, increasing the characteristic impedance reduces the transmission line efficiency linearly.

\section{REFERENCES}

[1] J. Arrillaga and N. R. Watson, "Computer Modelling of Electrical Power Systems", 2nd ed., Wiley, 2001.

[2] H. Saadat, "Power System Analysis", 3rd ed., PSA Publishing LLC, 2011.

[3] J. D. Glover, T. Overbye, M. S. Sarma, "Power System Analysis and Design", Cengage Learning, 2016.

[4] J. Grainger and W. D. Stevenson, "Power System Analysis", McGraw-Hill, 1994.

[5] Z. Huafeng, Z. Xuanyu, S. Di, Z. Huashi, J. Chaoyang, " PMU-based Transmission Line Parameter Identification at China Southern Power Grid", International Journal of Applied Power Engineering (IJAPE), vol. 3, no. 3, December 2014, pp. 190-198, ISSN: 2252-8792.

[6] V. K. Mehta and R. Mehta, "Principles of Power System", Revised 4th ed., S. Chand, 2008.

[7] D. Issicaba and J. Coelho, "Rotational Load Flow Method for Radial Distribution Systems", International Journal of Electrical and Computer Engineering (IJECE), vol. 6, no. 3, June 2016, pp. 1344-1352, ISSN: 2088-8708.

[8] S. Nawaz, A. Bansal and M.P. Sharma, "An Analytical Approach for DG Placement in Reconfigured Distribution Networks", International Journal of Applied Power Engineering (IJAPE), vol. 5, no. 3, December 2016, pp. 137-143, ISSN: 2252-8792.

[9] K. Nithiyananthan and Umasankar, "Environment Friendly Voltage Up-gradation Model for Distribution Power Systems", International Journal of Electrical and Computer Engineering (IJECE), vol. 6, no. 6, December 2016, pp. 2516-2525, ISSN: 2088-8708.

[10] N. Tleis, "Power Systems Modelling and Fault Analysis Theory and Practice", Newnes, 2008.

[11] D. Das, "Electrical Power Systems", New Age International (P) Ltd, 2006.

[12] M. Taleb, M. J. Ditto and T. Bouthiba, "Performance of short transmission lines models," 2006 IEEE GCC Conference (GCC), Manama, 2006, pp. 1-7.

[13] ABB Power T\&D Company Inc., "Electrical Transmission and Distribution Reference Handbook", ABB, 1997.

[14] N. Mohan, " Electric Power Systems: A First Course", Wiley, 2012.

\section{BIOGRAPHY OF AUTHOR}

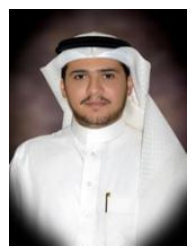

Dr. Muhyaddin Jamal H Rawa was born in Makkah, Saudi Arabia on August 03 1977. He received a BSc degree in Electrical and Computer Engineering from Umm Al-Qura University, an MSc degree in Electrical and Computer Engineering, Power and Machines, from King Abdulaziz University and a $\mathrm{PhD}$ in electrical and electronic engineering from the University of Nottingham in 2000, 2008 and 2014, respectively. He has more than 6 years experience in Saudi Electricity Company before joining the Department of Electrical and Computer Engineering at King Abdulaziz Univeristy as a Lecturer in 2008. Currently, he is an assistant professor at King Abdulaziz Univeristy. His research interest includes power quality, system reliability and smart grids. Dr Muhyaddin is a member of IEEE. 